\title{
Charge transfer at large scattering angles in the strong-potential Born approximation
}

\author{
Per A Amundsen $\dagger$ and D H Jakubaßa-Amundsen $\ddagger$ \\ $\dagger$ NORDITA, Blegdamsvej 17, DK-2100 København $\emptyset$, Denmark \\ $\ddagger$ Physik-Department, Technische Universität München, D-8046 Garching, West Germany
}

Received 24 March 1983, in final form 8 February 1984

\begin{abstract}
We have calculated the $1 \mathrm{~s}-1 \mathrm{~s}$ charge transfer probability for large projectile scattering angles in asymmetric ion-atom collisions, using the strong-potential Born approximation and including the effect of recoil on the projectile states non-perturbatively. A significant angular dependence is found. Numerical results for $1 \mathrm{~s}-1 \mathrm{~s}$ capture in $0.3-$ $20 \mathrm{MeV}$ proton impact on $\mathrm{C}$ and $\mathrm{Ne}$ are presented, showing good agreement with recent experimental results.
\end{abstract}

\section{Introduction}

In recent years there has been much interest in the theory of electron capture in asymmetric ion-atom collisions at intermediate and high energies. This interest is to a large extent due to the realisation that a systematic perturbative approach, which has become known as the strong-potential Born (SPB) approximation, after all is possible for such systems (Macek and Shakeshaft 1980, Jakubaßa-Amundsen and Amundsen 1980, Macek and Alston 1982). Unfortunately, this theory itself is not easy to evaluate exactly, but it does give a firm foundation for further approximations. It is in particular closely related to the impulse approximation (IA, McDowell 1961, Briggs 1977), and the most important quantitative SPB results obtained to date are indeed the large corrections to the total charge transfer cross sections at intermediate collision energies, compared with the IA (Macek and Taulbjerg 1981, Jakubaßa-Amundsen and Amundsen 1981). On the other hand, the difference in the impact parameter dependence between the two approximations is not very large in situations where a straight-line internuclear trajectory is a reasonable description.

Recently, measurements of charge transfer at large projectile scattering angles have been reported by Horsdal Pedersen et al $(1982 \mathrm{a}, \mathrm{b})$. When compared with the IA predictions (Amundsen and Jakubaßa-Amundsen 1982, hereafter referred to as AJA), theory and experiment could only be reconciled if it was assumed that transfer to projectile states of large angular momenta is important. The same conclusion follows from an analysis based on other simple theories, such as the Brinkman-Kramers approximation. However, other experimental evidence indicates that such transitions are not very important.

A qualitative model for resolving this problem has been proposed by Horsdal Pedersen and Rasmussen (1983). They argued that there could be an important amplitude present due to capture after the nuclear scattering of electrons that have 
been excited on the incoming part of the internuclear trajectory. Such an amplitude is not present in the IA, where the excitation and the capture are simultaneous, but it is present in the SPB, except for forward scattering, as shown in AJA. On the other hand, Kocbach and Briggs (1983) have pointed out that the influence of recoil on the projectile states is so strong that it generally cannot be treated perturbatively, as was implied in AJA. They showed that a non-perturbative treatment of this recoil gives rise to an angular dependence even for $1 \mathrm{~s}-1 \mathrm{~s}$ capture in the IA.

In the present paper we shall therefore investigate the SPB theory for charge transfer at large projectile scattering angles, including a non-perturbative treatment of recoil. In the next section we discuss the general theory, while in $\S 3$ we calculate the SPB amplitude for $1 \mathrm{~s}-1 \mathrm{~s}$ capture in the zero impact parameter approximation of Ciocchetti and Molinari (1965) with Briggs' (1977) peaking approximation, using hydrogenic wavefunctions. In $\S 4$ numerical results for proton impact on $\mathrm{C}$ and $\mathrm{Ne}$ are given, and the conclusions are drawn in $\S 5$. Atomic units $\left(e=\hbar=m_{\mathrm{e}}=1\right)$ are used throughout, and so are the abbreviations $\boldsymbol{a}=|\boldsymbol{a}|$ and $\hat{\boldsymbol{a}}=\boldsymbol{a} / \boldsymbol{a}$ for any vector $\boldsymbol{a}$.

\section{General considerations}

We consider transfer of an electron from a heavy target atom with an effective one-electron potential $V_{\mathrm{T}}$-the strong potential-to a light projectile associated with a potential $V_{\mathrm{p}}$. We shall work in the semiclassical picture, describing the internuclear motion by a classical path $\boldsymbol{R}(t)$. Following Dettmann (1971), the exact transfer amplitude as described in a target-fixed coordinate system can be written (in prior form):

$$
\begin{aligned}
& a_{f i}=-\mathrm{i} \int_{-\infty}^{\infty} \mathrm{d} t\left\langle\Psi^{(-)}(t)\left|V_{\mathrm{P}}(\boldsymbol{r}-\boldsymbol{R}(t))+V_{\mathrm{R}}(t)\right| i(t)\right\rangle^{\mathrm{T}} \\
& V_{\mathrm{R}}=\frac{1}{M_{T}} \frac{\partial V_{\mathrm{N}}}{\partial R} \hat{\boldsymbol{R}} \cdot \boldsymbol{r}=-\frac{M_{\mathrm{P}}}{M_{\mathrm{P}}+M_{\mathrm{T}}} \ddot{\boldsymbol{R}} \cdot \boldsymbol{r} .
\end{aligned}
$$

Here $|i(t)\rangle^{\mathrm{T}}=|i\rangle^{\mathrm{T}} \exp \left(-\mathrm{i} E_{i}^{\mathrm{T}} t\right)$ is the initial target eigenstate (energy $\left.E_{i}^{\mathrm{T}}\right),\left|\Psi^{(-)}(t)\right\rangle$ is an exact scattering solution of the total system which asymptotically develops into a prescribed projectile state, while $V_{\mathrm{R}}$ is the recoil potential, present since the target system is non-inertial, with $V_{\mathrm{N}}, M_{\mathrm{P}}$ and $M_{\mathrm{T}}$ the (central) nuclear potential and the projectile and target masses, respectively.

The SPB approximation is obtained from equation (2.1) by a systematic expansion of $\left|\Psi^{(-)}(t)\right\rangle$ in terms of the weak potential, which we shall take to be $V_{\mathrm{P}}+V_{\mathrm{R}}$, and retaining only the lowest order term. The role of $V_{\mathrm{R}}$ in this approximation will be to cause excitation of the target atom, like $V_{\mathrm{P}}$, and we shall for simplicity call this effect target recoil. But, as already stated, while the effect of the recoil on the target wavefunctions is mostly small enough for a perturbative treatment, its effect on an electron bound to the projectile is much stronger. This is both because the binding is much weaker and because the recoil as seen in the projectile system is a factor $\left(-M_{\mathrm{T}} / M_{\mathrm{P}}\right)$ stronger than given by $V_{\mathrm{R}} \dagger$. Thus the effect of recoil on $\left|\Psi^{(-)}(t)\right\rangle$, which we for

† There are some unfortunate errors in Jakubaßa-Amundsen and Amundsen (1980), as a factor $\left(-M_{2} / M_{1}\right)$ has been left out in front of $V_{\mathrm{R}}$ in equation (2.6) and also a term $-\ddot{\boldsymbol{R}} \cdot \boldsymbol{r}$ should be added to $V_{1}$ in equation (2.7) of that paper. These errors have propagated into AJA, where $-\ddot{R} \cdot \boldsymbol{r}$ should be added to $V_{1}$ in front of $\left|\psi_{f}^{\mathrm{P}}\left(t^{\prime}\right)\right\rangle$ in equation (2.6). These errors are of no consequence in a lowest order perturbative treatment of recoil, but affect one's assessment of the validity of such a treatment. 
convenience shall call projectile recoil, cannot be treated perturbatively. It is enough, however, to include it exactly in the absence of the target potential, since we can use the SPB expansion to also include the latter to all orders, and the remaining correction will then indeed turn out to be proportional to $V_{\mathrm{P}}+V_{\mathrm{R}}$ (see equation (2.3) below).

We have found no useful form of the solution for the projectile Schrödinger equation including recoil for a general $\boldsymbol{R}(t)$, but in the special case of a Ciocchetti-Molinari 'broken-line' path the acceleration is just $\ddot{R}(t)=\Delta v \delta(t)$, so that the sudden (Magnus) approximation becomes exact, and the appropriate scattering solution in the projectile rest frame is:

$$
\begin{gathered}
\left|\psi_{k}^{(-)}(t)\right\rangle^{\mathrm{P}}=\exp \left(-\mathrm{i} H_{\mathrm{P}} t\right) \exp \left\{\mathrm{i}\left[M_{\mathrm{T}} /\left(M_{\mathrm{P}}+M_{\mathrm{T}}\right)\right] \theta(-t) \boldsymbol{r} \cdot \Delta v\right\}|k\rangle^{\mathrm{P}} \\
=\sum_{n} \exp \left(-\mathrm{i} E_{n}^{\mathrm{P}} t\right)\left(W_{n k} \theta(-t)+\delta_{n k} \theta(t)\right)|n\rangle^{\mathrm{P}} \\
W_{n k}={ }^{\mathrm{P}}\left\langle n\left|\exp \left\{\mathrm{i}\left[M_{\mathrm{T}} /\left(M_{\mathrm{P}}+M_{\mathrm{T}}\right)\right] \boldsymbol{r} \cdot \Delta v\right\}\right| k\right\rangle^{\mathrm{P}} \\
\Delta v=\dot{\boldsymbol{R}}(\infty)-\dot{\boldsymbol{R}}(-\infty)
\end{gathered}
$$

where $|n\rangle^{\mathbf{P}}$ is a projectile eigenstate (of $H_{\mathrm{p}}$ with eigenvalue $E_{n}^{\mathrm{P}}$ ) and $\theta$ is the unit step function.

Starting with $\left|\psi_{k}^{(-)}(t)\right\rangle^{\mathrm{P}}$ (instead of just $|k\rangle^{\mathrm{P}}$ ) one finds, repeating with slight modifications the arguments of AJA, that $\left|\Psi^{(-)}(t)\right\rangle$ can be found from the equations:

$$
\begin{aligned}
& |\psi(t)\rangle=\left|\psi_{f}^{(-)}(t)\right\rangle+\int_{-\infty}^{\infty} \mathrm{d} t^{\prime} G_{0}^{(-)}\left(t, t^{\prime}\right) V_{\mathrm{T}}\left|\psi\left(t^{\prime}\right)\right\rangle \\
& \left|\Psi^{(-)}(t)\right\rangle=|\psi(t)\rangle+\int_{-\infty}^{\infty} \mathrm{d} t^{\prime} G_{\mathrm{T}}^{(-)}\left(t, t^{\prime}\right)\left(V_{\mathrm{P}}\left(\boldsymbol{r}-\boldsymbol{R}\left(t^{\prime}\right)\right)+V_{\mathrm{R}}\left(t^{\prime}\right)\right)\left(\left|\Psi^{(-)}\left(t^{\prime}\right)\right\rangle-\left|\psi_{f}^{(-)}\left(t^{\prime}\right)\right\rangle\right)
\end{aligned}
$$

where $G_{0}$ and $G_{\mathrm{T}}$ are free and target (time-dependent) Green's functions, respectively, and $\left|\psi_{f}^{(-)}(t)\right\rangle$ is $\left|\psi_{f}^{(-)}(t)\right\rangle^{\mathrm{P}}$ transformed to the target system. From equation (2.3) one can generate the SPB expansion for $\left|\Psi^{(-)}(t)\right\rangle$ in powers of our chosen weak potential, $V_{\mathrm{P}}+V_{\mathrm{R}}$. This expansion satisfies the correct boundary conditions termwise, and the lowest order solution is just $|\psi(t)\rangle$. Again following AJA, we can then solve equation (2.3a) exactly, and inserting the result into equation (2.1) we find:

$$
\begin{aligned}
a_{f i}=\frac{1}{2 \pi \mathrm{i}} \int_{-\infty}^{\infty} & \mathrm{d} t \\
& \left.+\int_{0}^{\infty} \mathrm{d} \boldsymbol{k} \int_{-\infty}^{\infty} \mathrm{d} t^{\prime} \varphi_{f}^{\mathrm{P}^{*}}\left(\boldsymbol{k}-\dot{\boldsymbol{R}}\left(t_{-\infty}^{0}\right)\right) \exp \left[\mathrm{i}\left(\varepsilon_{f}\left(t^{\prime}\right)-\omega t^{\prime}\right)\right]\right) \\
& \times \exp \left[\mathrm{i}\left(\omega-E_{i}^{\mathrm{T}}\right) t\right]^{\mathrm{T}}\left\langle\boldsymbol{k}, \omega\left|V_{\mathrm{P}}(\boldsymbol{r}-\boldsymbol{R}(t))+V_{\mathrm{R}}(t)\right| i\right\rangle^{\mathrm{T}}
\end{aligned}
$$

where $\varphi_{n}^{\mathrm{P}}$ is a momentum space projectile wavefunction, and $|\boldsymbol{k}, \omega\rangle^{\mathrm{T}}$ an off-shell target continuum state of momentum $\boldsymbol{k}$ and energy $\omega-\mathrm{i} \varepsilon$. The infinitesimally small imaginary part of $\omega$ ensures that the correct boundary conditions on $\left|\Psi^{(-)}(t)\right\rangle$ are preserved in $a_{f i}$. The effective projectile energy phase as seen in the target frame is

$\varepsilon_{n}(t)=E_{n}^{\mathrm{P}} t+\boldsymbol{k} \cdot \boldsymbol{R}(t)-\frac{1}{2} \frac{M_{\mathrm{T}}-M_{\mathrm{P}}}{M_{\mathrm{T}}+M_{\mathrm{P}}} \int_{0}^{t} \dot{R}^{2}(t) \mathrm{d} t-\frac{M_{\mathrm{P}}}{M_{\mathrm{T}}+M_{\mathrm{P}}} \dot{\boldsymbol{R}}(t) \cdot \boldsymbol{R}(t)$.

If $\boldsymbol{R}(t)$ is not a broken line path, but reasonably well approximated by one, the 
corrections to the recoil contributions occur only in the next to the leading order in the perturbation expansion, and thus do not contribute to equation (2.4).

The above expression for $a_{f i}$ has a straightforward physical interpretation: the projectile potential (or the target recoil) causes a transition between the target states $|i\rangle^{\mathbf{T}}$ and $|\boldsymbol{k}, \omega\rangle^{\mathbf{\top}}$ at time $t$. Subsequently, at time $t^{\prime}$, the electron finds itself in the moving projectile state $\varphi_{n}^{\mathrm{P}}$, and is captured. If $t^{\prime}>0$, i.e. after the nuclear scattering, the electron will follow the projectile out of the collision, remaining in the state in which it was captured, i.e. $n=f$. But if $t^{\prime}<0$, an electron captured in an arbitrary state has a probability of being 'shaken over' into the prescribed final state by the strong recoil caused by the nuclear collision at $t=0$. This is described by $W_{n f}$. The i $\varepsilon$ prescription on $\omega$ ensures that indeed $t^{\prime}>t$. Also, since the electron only remains a finite time in the state $|\boldsymbol{k}, \omega\rangle^{\mathrm{T}}$, the energy-time uncertainty relation tells that it should have a distribution in energy.

The IA is obtained by replacing the off-shell state $|\boldsymbol{k}, \omega\rangle^{\mathrm{T}}$ in equation (2.4) by its on-shell counterpart $|\boldsymbol{k}\rangle^{\mathrm{T}}\left(\omega=\frac{1}{2} k^{2}\right)$. This makes the $\omega$ integral trivial, yielding $2 \pi \delta\left(t-t^{\prime}\right)$. Thus the time delay between the excitation and the capture process disappears, so that the amplitude for excitation before the nuclear scattering and capture after vanishes. The same is true if one takes $|\boldsymbol{k}, \omega\rangle^{\mathrm{T}}$ at a fixed off-shell value. The resulting version of the IA is not identical to the one in AJA, due to the presence of projectile recoil. The AJA formula is obtained by a further replacement of $W_{n f}$ by $\delta_{n f}$ If one does this, the predicted scattering angle $(\vartheta)$ dependence of the capture probability becomes very simple in structure, in particular for s-s capture no angular dependence is predicted. If the projectile recoil is retained, this is no longer true (Kocbach and Briggs 1983).

There is thus the possibility of a qualitative difference between the predictions of the SPB approximation and the IA for the dependence of the capture probabilities on $\vartheta$, due to the time delay between excitation and capture. It should be noted that for forward scattering $(\vartheta=0)$ this delay vanishes. This is because the energy transfer becomes related to the momentum transfer, so that the (off-shell) energy of the state $|\boldsymbol{k}, \omega\rangle^{\mathrm{T}}$ is no longer unsharp, but has the value $\omega=E_{f}(\boldsymbol{k})=E_{f}^{\mathrm{P}}+\boldsymbol{k} \cdot \boldsymbol{v}-\frac{1}{2} v^{2}$. This relation between energy transfer and momentum transfer can be traced back to energy momentum conservation in a fully quantal description of the collision process.

In order to simplify the SPB amplitude (equation (2.4)) we note that it is only weakly dependent on the value of $E_{n}^{\mathrm{P}}$, which enters through $\varepsilon_{n}(t)$, for asymmetric systems. This is because $\left|E_{n}^{\mathrm{P}}\right| \ll\left|E_{i}^{\mathrm{T}}\right|$ for all $n$ which contribute appreciably to the amplitude, and it is $E_{i}^{\mathrm{T}}$ that sets the natural scale for the energy phases in equation (2.4). If we replace $E_{n}^{\mathrm{P}}$ by some mean value, $\bar{E}^{\mathrm{P}}$, we can use the completeness of the set $\left\{|n\rangle^{\mathrm{P}}\right\}$ to obtain:

$\sum_{n} \varphi_{n}^{\mathrm{P}}(\boldsymbol{k}-\dot{\boldsymbol{R}}(t))\left[W_{n f} \theta(-t)+\delta_{n f} \theta(t)\right]=\varphi_{f}^{\mathrm{P}}\left(\boldsymbol{k}-\dot{\boldsymbol{R}}(t)-\frac{M_{\mathrm{T}}}{M_{\mathrm{T}}+M_{\mathrm{P}}} \Delta v \theta(-t)\right)$.

Furthermore, as long as $\varphi_{f}^{\mathrm{P}}$ represents a bound state, it can be expanded in a uniformly convergent expansion after $M_{\mathrm{P}} /\left(M_{\mathrm{P}}+M_{\mathrm{T}}\right) \leqslant Z_{\mathrm{P}} / Z_{\mathrm{T}}$. Retaining only the zeroth-order contribution to this expansion is consistent with the first-order treatment of the projectile field in the SPB. Thus we find

$$
\begin{aligned}
a_{f i}=\frac{1}{2 \pi \mathrm{i}} \int_{-\infty}^{\infty} \mathrm{d} t \int \mathrm{d} \boldsymbol{k} \int_{-\infty}^{\infty} \mathrm{d} \omega \int_{-\infty}^{\infty} \mathrm{d} \boldsymbol{t}^{\prime} \varphi_{f}^{\mathrm{P}^{*}}\left(\boldsymbol{k}-\dot{\boldsymbol{R}}\left(t^{\prime}\right)-\Delta \boldsymbol{v} \theta\left(-\boldsymbol{t}^{\prime}\right)\right) \\
\quad \times \exp \left[\mathrm{i}\left(\bar{\varepsilon}\left(t^{\prime}\right)-\omega t^{\prime}\right)\right] \exp \left[\mathrm{i}\left(\omega-E_{i}^{\mathrm{T}}\right) t\right]^{\mathrm{T}}\left\langle\boldsymbol{k}, \omega\left|V_{\mathrm{P}}+V_{\mathrm{R}}\right| i\right\rangle^{\mathrm{T}}
\end{aligned}
$$


where $\bar{\varepsilon}\left(t^{\prime}\right)$ follows from equation (2.5) with $E_{n}^{\mathrm{P}}$ replaced by $\bar{E}^{\mathrm{P}}$. Note that for the 'broken-line' path the argument of $\varphi_{f}^{\mathrm{P}}$ is just shifted by the outgoing momentum, $\dot{\boldsymbol{R}}(\infty)$ even for $t^{\prime}<0$. This implies that for asymmetric systems the projectile recoil induces no extra isotope effects in the transfer amplitude.

As for the validity of the 'broken-line' path we shall just note the following: in general this is a good approximation provided $\ddot{\boldsymbol{R}}(t) \neq 0$ only for a short time compared with the typical timescale of the active electron. For excitation of the target, we have that a Rutherford trajectory, $\boldsymbol{R}_{\mathrm{R}}(t)$ is adequately represented by the 'broken-line' path provided $q^{\mathrm{T}} R_{\mathrm{R}}(0) \ll 1$, where the typical momentum transfer $q$ in the present case is $q^{\mathrm{T}}=\left(\left|E_{i}^{\mathrm{T}}\right|-\left|E_{f}^{\mathrm{P}}\right|\right) / v+\frac{1}{2} v(v=|\dot{\boldsymbol{R}}( \pm \infty)|)$. This condition can be violated for capture from inner shells in heavy target atoms. For the capture process, the timescale is set by the projectile, and the sudden approximation should be valid provided $q^{\mathrm{P}} R_{\mathrm{R}}(0) \ll 1$, where $q^{\mathrm{P}}=\max \left(\frac{1}{2} Z_{\mathrm{P}}^{2}, \frac{1}{2}|\Delta v|^{2}\right) / v$, since the collision time is approximately $R_{R}(0) / v$. This condition is in general weaker than the one above for asymmetric collisions. Thus, for electron capture by light ions from inner shells of heavy atoms the broken-line path is sufficient for the capture part of equation (2.4), but should be replaced by a Rutherford trajectory in the ionisation matrix element for very slow collisions. But for situations of present experimental interest this is an unnecessary complication.

\section{1s-1s capture}

In this section we shall assume $V_{\mathrm{P}}$ and $V_{\mathrm{T}}$ to be Coulomb potentials of charges $Z_{\mathrm{P}}$ and $Z_{\mathrm{T}}$, respectively. In this case the off-shell Coulomb wavefunctions, $|\boldsymbol{k}, \omega\rangle^{\mathrm{T}}$ are known exactly (Chen and Chen 1972 and references therein), but not in a form very suitable for further manipulations. As in all SPB calculations so far we shall therefore rely on the approximate result (Macek and Taulbjerg 1981)

$$
\begin{aligned}
& |\boldsymbol{k}, \omega\rangle^{\mathrm{T}} \underset{\omega=k^{2} / 2}{\simeq} \Gamma(1-\mathrm{i} \eta) \exp (-\pi \eta / 2)\left(\frac{\omega-\mathrm{i} \varepsilon-k^{2} / 2}{4 \omega}\right)^{\mathrm{i} \eta}|\boldsymbol{k}\rangle^{\mathrm{T}} \\
& \eta=Z_{\mathrm{T}} / k
\end{aligned}
$$

where we have written the imaginary part of $\omega,(-i \varepsilon)$ where its presence is crucial. Since this approximation is only valid for $\omega \rightarrow \frac{1}{2} k^{2}$, we replace the denominator $4 \omega$ in this expression by $2 k^{2}$, which is also in the spirit of the peaking approximation that we shall make further on. The $\omega$ integral in equation (2.4) is then easily done.

As argued in the previous section, for the present purposes the Rutherford trajectory is adequately approximated by a zero impact parameter broken-line path:

$$
\boldsymbol{R}(t) \equiv v_{\mp} t=v t(\mp \sin \vartheta / 2,0, \cos \vartheta / 2) \quad t \lessgtr 0 .
$$

The energy phase $\bar{\varepsilon}\left(t^{\prime}\right)$ then becomes, from equation (2.5)

$$
\begin{aligned}
& \bar{\varepsilon}(t)=\Omega_{\mp} t \quad t \lessgtr 0 \\
& \Omega_{\mp}=\bar{E}^{\mathrm{P}}+\boldsymbol{k} \cdot v_{\mp}-\frac{1}{2} v^{2}
\end{aligned}
$$

and writing $\varphi_{f}^{\mathrm{P}}=\varphi_{f}^{\mathrm{P}}\left(\boldsymbol{k}-\boldsymbol{v}_{+}\right)$where $\varphi_{f}^{\mathrm{P}}$ can be taken to be real, the $t^{\prime}$ integral can be carried out by splitting the $t$ integral according to $t \lessgtr 0$, and using the standard integral representation of the incomplete gamma function (Abramowitz and Stegun 1965)

$$
\gamma(\mathrm{i} \eta, \mathrm{i} y t)=(\mathrm{i} y)^{\mathrm{i} \eta} \int_{0}^{t} \exp (-\mathrm{i} y \tau) \tau^{\mathrm{i} \eta-1} \mathrm{~d} \tau .
$$


One finds

$$
\begin{aligned}
a_{f}=\int \mathrm{d} k \eta\left(2 k^{2}\right)^{\mathrm{i} \eta} & \langle k|\left[\int _ { - \infty } ^ { 0 } \mathrm { d } t \left(\varphi_{f}^{\mathrm{P}} \frac{\gamma\left(\mathrm{i} \eta, \mathrm{i}\left(\Omega_{-}-\frac{1}{2} k^{2}+\mathrm{i} \varepsilon\right) t\right)}{\left[-\mathrm{i}\left(\Omega_{--}-\frac{1}{2} k^{2}+\mathrm{i} \varepsilon\right)\right]^{\mathrm{i} \eta}} \exp \left(\mathrm{i} \Omega_{-} t\right)\right.\right. \\
& \left.+\varphi_{f}^{\mathrm{P}} \frac{\Gamma(\mathrm{i} \eta)-\gamma\left(\mathrm{i} \eta, \mathrm{i}\left(\Omega_{+}-\frac{1}{2} k^{2}+\mathrm{i} \varepsilon\right) t\right)}{\left[-\mathrm{i}\left(\Omega_{+}-\frac{1}{2} k^{2}+\mathrm{i} \varepsilon\right)\right]^{\mathrm{i} \eta}} \exp \left(\mathrm{i} \Omega_{+} t\right)\right) \\
& \left.+\int_{0}^{\infty} \mathrm{d} t \varphi_{f}^{\mathrm{P}} \exp \left(\mathrm{i} \Omega_{+} t\right) \frac{\Gamma(\mathrm{i} \eta)}{\left[-\mathrm{i}\left(\Omega_{+}-\frac{1}{2} k^{2}+\mathrm{i} \varepsilon\right)\right]^{\mathrm{i} \eta}}\right]\left(V_{\mathrm{P}}+V_{\mathrm{R}}\right) \exp \left(-\mathrm{i} E_{i}^{\mathrm{T}} t\right)|i\rangle^{\mathrm{T}}
\end{aligned}
$$

For notational convenience we shall suppress the target recoil term in the following, it can be handled in a manner analogous to the potential term, indeed the mathematical manipulations become somewhat simpler (see AJA). We shall return to it the end of this section. Introducing the Fourier transform of $V_{\mathrm{P}}$ and noticing that from equation (3.4) we have with an interchange of the order of integrations $(\operatorname{Im} y<0)$ :

$$
\int_{0}^{\infty} \mathrm{d} t \exp (-\mathrm{i} x t) \gamma(\mathrm{i} \eta, \mathrm{i} y t)=\Gamma(\mathrm{i} \eta)\left[\pi \delta(x)+\frac{1}{\mathrm{i} x}\left(1+\frac{x}{y}\right)^{-\mathrm{i} \eta}\right]
$$

the $t$-integral is readily done, yielding for the transition amplitude

$$
\begin{aligned}
& a_{f i}=\frac{\mathrm{i} Z_{\mathrm{P}}}{2 \pi^{2}} \int \mathrm{d} \boldsymbol{k}\left(2 k^{2}\right)^{\mathrm{i} \eta} \exp (-\pi \eta / 2) \Gamma(1+\mathrm{i} \eta) \int \frac{\mathrm{d} \boldsymbol{s}^{\mathrm{T}}}{\boldsymbol{s}^{2}}\langle\boldsymbol{k}|\exp (\mathrm{i} \boldsymbol{s} \cdot \boldsymbol{r})| \mathrm{i}\rangle^{\mathrm{T}} \varphi_{f}^{\mathrm{P}} \\
& \times\left(\pi \frac{\delta\left(\Omega_{-}-E_{\mathrm{i}}^{\mathrm{T}}-\boldsymbol{s} \cdot \boldsymbol{v}_{-}\right)}{\left(\Omega_{-}-\frac{1}{2} k^{2}+\mathrm{i} \varepsilon\right)^{\mathrm{i} \eta}}+\pi \frac{\delta\left(\Omega_{+}-E_{\mathrm{i}}^{\mathrm{T}}-s \cdot v_{+}\right)}{\left(\Omega_{+}-\frac{1}{2} k^{2}+\mathrm{i} \varepsilon\right)^{\mathrm{i} \eta}}\right. \\
& +\frac{\left(E_{\mathrm{i}}^{\mathrm{T}}-\frac{1}{2} k^{2}+\boldsymbol{s} \cdot \boldsymbol{v}_{-}+\mathrm{i} \varepsilon\right)^{-\mathrm{i} \eta}}{\mathrm{i}\left(\Omega_{-}-E_{\mathrm{i}}^{\mathrm{T}}-\boldsymbol{s} \cdot \boldsymbol{v}_{-}\right)}-\frac{\left(E_{\mathrm{i}}^{\mathrm{T}}-\frac{1}{2} k^{2}+\boldsymbol{s} \cdot \boldsymbol{v}_{-}+\mathrm{i} \varepsilon\right)^{-\mathrm{i} \eta}}{\mathrm{i}\left(\Omega_{+}-E_{\mathrm{i}}^{\mathrm{T}}-\boldsymbol{s} \cdot \boldsymbol{v}_{-}\right)} \\
& \left.+\left(\Omega_{+}-\frac{1}{2} k^{2}+\mathrm{i} \varepsilon\right)^{-\mathrm{i} \eta} \frac{1}{\mathrm{i}}\left[\left(\Omega_{+}-E_{\mathrm{i}}^{\mathrm{T}}-\boldsymbol{s} \cdot v_{-}\right)^{-1}-\left(\Omega_{+}-E_{\mathrm{i}}^{\mathrm{T}}-s \cdot v_{+}\right)^{-1}\right]\right) .
\end{aligned}
$$

Here the principal value of the singular integrals is implied where no explicit dependence on $\varepsilon$ is indicated. So far, the results are valid for arbitrary projectile and target wavefunctions.

Since the difference between the SPB and the IA results occurs even for $1 \mathrm{~s}-1 \mathrm{~s}$ transitions, we shall restrict ourselves to these. In order to isolate the scattering angle dependence of the amplitudes, one can exploit that ${ }^{\mathrm{T}}\langle\boldsymbol{k}, \omega|\exp (\mathrm{is} \cdot \boldsymbol{r})| 1 \mathrm{~s}\rangle^{\mathrm{T}}$ is invariant under symmetry operations such as a simultaneous reflection or rotation of $s$ and $\boldsymbol{k}$, as $\hat{\boldsymbol{s}}$ and $\hat{\boldsymbol{k}}$ enter only through $\boldsymbol{k} \cdot \boldsymbol{s}$.

We use the explicit expressions for the hydrogenic $1 \mathrm{~s}$ state $\varphi_{1 \mathrm{~s}}^{\mathrm{P}}=$ $2^{3 / 2} Z_{\mathrm{P}}^{5 / 2} /\left\{\pi\left[Z_{\mathrm{P}}^{2}+\left(k-v_{+}\right)^{2}\right]^{2}\right\}$, choose $\bar{E}^{\mathrm{P}}=-\frac{1}{2} Z_{\mathrm{P}}^{2}$ for definiteness and apply the reflection $k_{x}, s_{x} \rightarrow-k_{x},-s_{x}$ to all terms but the first in equation (3.7), in order to keep the arguments of the $\delta$ functions as simple as possible. A further rotation of $\boldsymbol{k}$ and $\boldsymbol{s}$ through an angle $\vartheta / 2$, so that $\boldsymbol{k} \cdot \boldsymbol{v}_{-} \rightarrow k_{z} v$, eliminates the dependence on $\vartheta$ from all terms of (3.6) except those which contain $v_{-}$. With these operations, we arrive at

$a_{f i}=\frac{\mathrm{i} Z_{\mathrm{P}}^{7 / 2} \sqrt{2}}{\pi^{3}} \int \mathrm{d} \boldsymbol{k}\left(2 k^{2}\right)^{\mathrm{i} \eta} \exp (-\pi \eta / 2) \Gamma(1+\mathrm{i} \eta) \int \frac{\mathrm{d} s^{2}}{s^{2}}\langle\boldsymbol{k}|\exp (\mathrm{i} s \cdot \boldsymbol{r})| \mathrm{i}\rangle^{\mathrm{T}}$ 


$$
\begin{aligned}
& \times\left[\frac { 2 ^ { \mathrm { i } \eta } \operatorname { e x p } ( \pi \eta ) } { [ Z _ { \mathrm { P } } ^ { 2 } + ( k - v \boldsymbol { e } _ { z } ) ^ { 2 } ] ^ { 2 + \mathrm { i } \eta } } \left\{\pi \delta\left[\Delta E+\left(k_{z}-s_{z}\right) v-\frac{1}{2} v^{2}\right]\right.\right. \\
& +\mathrm{i}\left[\Delta E+\left(k_{z}-s_{z}\right) v-\frac{1}{2} v^{2}\right]^{-1} \\
& \left.-\mathrm{i}\left[\Delta E-2 \tilde{s}_{x} v \sin \vartheta / 2+\left(k_{z}-s_{z}\right) v-\frac{1}{2} v^{2}\right]^{-1}\right\}-\mathrm{i}\left[Z_{\mathrm{P}}^{2}+\left(k-v \boldsymbol{e}_{z}\right)^{2}\right]^{-2} \\
& \times\left(\frac{\left.E_{\mathrm{i}}^{\mathrm{T}}-\frac{1}{2} k^{2}+\mathrm{i} \varepsilon+2 \tilde{s}_{x} v \sin \vartheta / 2+s_{z} v\right)^{-\mathrm{i} \eta}}{\Delta E+\left(k_{x} \sin \vartheta+k_{z} \cos \vartheta-2 \tilde{s}_{x} \sin \vartheta / 2\right) v-\frac{1}{2} v^{2}-s_{z} v}\right. \\
& \left.-\frac{\left(E_{\mathrm{i}}^{\mathrm{T}}-\frac{1}{2} k^{2}+\mathrm{i} \varepsilon+2 \tilde{s}_{x} v \sin \vartheta / 2+s_{z} v\right)^{-\mathrm{i} \eta}}{\Delta E-2 \tilde{s}_{x} v \sin \vartheta / 2+\left(k_{z}-s_{z}\right) v-\frac{1}{2} v^{2}}\right) \\
& \left.+\frac{2^{\mathrm{i} \eta} \exp (\pi \eta)}{\left[Z_{\mathrm{P}}^{2}+\left(k-\tilde{v}_{+}\right)^{2}\right]^{2}} \frac{\pi \delta\left(\Delta E+\left(k_{z}-s_{z}\right) v-\frac{1}{2} v^{2}\right)}{\left[Z_{\mathrm{P}}^{2}+\left(k-v e_{z}\right)^{2}\right]^{\mathrm{i} \eta}}\right]
\end{aligned}
$$

where

$$
\begin{aligned}
& \Delta E=\bar{E}^{\mathrm{P}}-E_{\mathrm{i}}^{\mathrm{T}} \\
& \tilde{s}_{x}=s_{x} \cos \vartheta / 2-s_{z} \sin \vartheta / 2 \quad \tilde{v}_{+}=v(\sin \vartheta, 0, \cos \vartheta)
\end{aligned}
$$

which implies $2 \tilde{s}_{x} \sin \vartheta / 2+s_{z}=s_{x} \sin \vartheta+s_{z} \cos \vartheta$.

For hydrogenic wavefunctions the target matrix element ${ }^{\mathrm{T}}\langle\boldsymbol{k}|\exp (\mathrm{is} \cdot \boldsymbol{r})| 1 \mathrm{~s}\rangle^{\mathrm{T}}$ is well known (McDowell and Coleman 1970):

$$
\begin{aligned}
& { }^{\mathrm{T}}\langle\boldsymbol{k}|\exp (\mathrm{is} \cdot \boldsymbol{r})| 1 \mathrm{~s}\rangle^{\mathrm{T}}=\frac{2^{3 / 2} Z_{\mathrm{T}}^{5 / 2}}{\pi} \exp (\pi \eta / 2) \Gamma(1-\mathrm{i} \eta) H_{0}(s, k, x) \\
& H_{0}(s, k, x)=\frac{\left[s^{2}-\left(k+\mathrm{i} Z_{\mathrm{T}}\right)^{2}\right]^{-\mathrm{i} \eta}}{\left(Z_{\mathrm{T}}^{2}+s^{2}+k^{2}-2 s k x\right)^{2-\mathrm{i} \eta}}\left(1-\mathrm{i} \eta+(1+\mathrm{i} \eta) \frac{Z_{\mathrm{T}}^{2}+s^{2}+k^{2}-2 k s x}{s^{2}-\left(k+\mathrm{i} Z_{\mathrm{T}}\right)^{2}}\right) \\
& x=\hat{\boldsymbol{k}} \cdot \hat{s} .
\end{aligned}
$$

A direct evaluation of equation (3.8) with this matrix element leads to rather complex expressions.

We shall circumvent this problem by applying the peaking approximation introduced by Briggs (1977), and used in most subsequent papers on the SPB approximation (Macek and Taulbjerg 1981, Macek and Alston 1982). This peaking relies on the fact that the final-state wavefunction $\varphi_{f}^{\mathrm{P}}(\boldsymbol{k}-\boldsymbol{v})$ is strongly peaked at $\boldsymbol{k}=\boldsymbol{v}$, so that the slowly varying parts of the integrand may be taken outside the $\boldsymbol{k}$ integral at this value of $\boldsymbol{k}$. It is crucial, however, not to use it for factors that are oscillating strongly for some value of $s$ or $k$, or in terms which after this approximation would become of order $Z_{\mathrm{p}}$. Thus, we replace $\boldsymbol{k}$ by $v \boldsymbol{e}_{z}$ everywhere except in the last term of (3.8), in $\left[Z_{\mathrm{P}}^{2}+\left(\boldsymbol{k}-\boldsymbol{v} \boldsymbol{e}_{z}\right)^{2}\right]^{\lambda}$ $(\lambda=2,2+\mathrm{i} \eta)$ and in the factors containing i $\varepsilon$. The last term, as it stands, is peaked at $\boldsymbol{k}=\tilde{\boldsymbol{v}}_{+}$, and correspondingly we replace $\boldsymbol{k}$ by $\tilde{\boldsymbol{v}}_{+}$instead of $v \boldsymbol{e}_{z}$. With these approximations, the $\boldsymbol{k}$ and $\varphi_{s}$ integrals can be carried out analytically. The details are given in the appendix, including a further non-crucial approximation to speed up the numerical calculations. Using the expressions of the appendix and $|\Gamma(1+\mathrm{i} \eta)|^{2}=\pi \eta / \sinh \pi \eta$, we finally arrive at the expression

$$
a_{f i}=\frac{16 \mathrm{i}\left(2 v^{2}\right)^{\mathrm{i} \eta} \eta\left(Z_{\mathrm{P}} Z_{\mathrm{T}}\right)^{5 / 2}}{1-\exp (-2 \pi \eta)}\left(a_{1}+a_{2}\right)
$$




$$
\begin{array}{rl}
a_{1}=\frac{\sqrt{\pi}}{v}\left(\frac{2}{Z_{\mathrm{P}}^{2}}\right)^{\mathrm{i} \eta} & \frac{\Gamma\left(\frac{1}{2}+\mathrm{i} \eta\right)}{\Gamma(2+\mathrm{i} \eta)} \int_{s_{\text {min }}}^{\infty} \frac{\mathrm{d} s}{s} H_{0}\left(s, v, x_{0}\right)+\frac{\mathrm{i} \sqrt{\pi}}{4 v \xi}\left(\frac{2}{Z_{\mathrm{P}}^{2}}\right)^{\mathrm{i} \eta} \frac{\Gamma\left(-\frac{1}{2}+\mathrm{i} \eta\right)}{\Gamma(1+\mathrm{i} \eta)} \\
& \times\left[\frac{1}{t_{+}}{ }_{2} F_{1}\left(1, \frac{3}{2} ; 1+\mathrm{i} \eta, 1+\frac{1}{t_{+}}\right)-\frac{1}{t_{-}}{ }_{2} F_{1}\left(1, \frac{3}{2} ; 1+\mathrm{i} \eta ; 1+\frac{1}{t_{-}}\right)\right] \\
& \times \int_{s_{\text {min }}}^{\infty} \frac{\mathrm{d} s}{s}\left[(1-\mathrm{i} \eta)\left[s^{2}-\left(v+\mathrm{i} Z_{\mathrm{T}}\right)^{2}\right]^{-\mathrm{i} \eta}\left(\lambda_{1}+\mu_{1}\right)^{-2+\mathrm{i} \eta}\right. \\
& \times{ }_{2} F_{1}\left(2-\mathrm{i} \eta, \frac{1}{2} ; 1 ; \frac{2 \mu_{1}}{\lambda_{1}+\mu_{1}}\right) \\
& +(1+\mathrm{i} \eta)\left[s^{2}-\left(v+\mathrm{i} Z_{\mathrm{T}}\right)^{2}\right]^{-\mathrm{i} \eta-1}\left(\lambda_{1}+\mu_{1}\right)^{-1+\mathrm{i} \eta} \\
& \left.\times{ }_{2} F_{1}\left(1-\mathrm{i} \eta, \frac{1}{2} ; 1 ; \frac{2 \mu_{1}}{\lambda_{1}+\mu_{1}}\right)\right] \\
a_{2}=\mathrm{i} \int_{0}^{\infty} \mathrm{d} s \int_{-1}^{1} & \mathrm{~d} x H_{0}(s, v, x)\left[\frac{1}{\sqrt{\pi}}\left(\frac{2}{Z_{\mathrm{p}}}\right)^{\mathrm{i} \eta} \frac{\Gamma\left(\frac{1}{2}+\mathrm{i} \eta\right)}{\Gamma(2+\mathrm{i} \eta)}\right. \\
& \times\left(\left(\Delta E-s v x+\frac{1}{2} v^{2}\right)^{-1}-\frac{\operatorname{sign} C \theta(|C|-B)}{\left.\left(C^{2}-B^{2}\right)^{1 / 2}\right)}\right. \\
& \left.+\frac{\exp (-\pi \eta)}{2 v} \sum_{j=0}^{1}(-1)^{j} \frac{\left(v-\mathrm{i} Z_{\mathrm{P}}\right) F_{j}\left(A_{-}\right)+\left(v+\mathrm{i} Z_{\mathrm{P}}\right) F_{j}\left(A_{+}\right)}{\left(C_{j}^{2}-B^{2}\right)^{1 / 2}}\right] \\
x_{0}=\frac{s_{\min _{0}}}{s} & s_{\min _{0}}=\frac{\Delta E+\frac{1}{2} v^{2}}{v}
\end{array}
$$

Here $a_{1}$ arises from the first and the last terms of equation (3.8), which correspond to the IA, corrected by the Macek-Taulbjerg (1981) factor, while $a_{2}$ contains all the other terms, including the dependence on the scattering angle through the variables $A_{ \pm}, B$ and $C_{i}$, which together with $\lambda_{1}, \mu_{1}$ and $x_{1}$ are defined in the appendix. For $\vartheta=0$, $a_{2}=0$, as it should.

The target recoil amplitude, $a_{f i}^{\mathrm{R}}$ must be added to the above amplitude. The evaluation is straightforward, as there is only the integral over momentum $\boldsymbol{k}$ to be carried out. This can easily be done if a similar rotation to the one leading to equation (3.8) is performed, and the Briggs peaking approximation used. One finds that there is only a contribution to $a_{f i}^{\mathrm{R}}$ when the capture takes place on the outgoing part of the trajectory $\left(t^{\prime}>0\right)$, the result is:

$$
\begin{aligned}
& a_{f i}^{\mathrm{R}}=C_{f i} \sin ^{2} \frac{\vartheta}{2} \\
& C_{f i}=-\frac{64 \sqrt{\pi}}{M_{\mathrm{P}}+M_{\mathrm{P}}} Z_{\mathrm{P}}^{3 / 2-2 \mathrm{i} \eta} Z_{\mathrm{T}}^{5 / 2} \frac{v^{2}\left(4 v^{2}\right)^{\mathrm{i} \eta}}{\left(Z_{\mathrm{T}}^{2}+v^{2}\right)^{2-\mathrm{i} \eta}} \frac{\eta(1-\mathrm{i} \eta)}{1-\exp (-2 \pi \eta)} \\
& \quad \times\left[-\left(v+\mathrm{i} Z_{\mathrm{T}}\right)^{2}\right]^{-\mathrm{i} \eta}\left(\frac{1+\mathrm{i} \eta}{\left(v+\mathrm{i} Z_{\mathrm{T}}\right)^{2}}-\frac{2-\mathrm{i} \eta}{Z_{\mathrm{T}}^{2}+v^{2}}\right) \frac{\Gamma\left(\frac{1}{2}+\mathrm{i} \eta\right)}{\Gamma(2+\mathrm{i} \eta)} .
\end{aligned}
$$

The SPB recoil term shows an angular dependence even for 1s-1s transfer, with its maximum in the backward direction. This is so because the recoil itself is largest for 
$180^{\circ}$ deflection and because there is a constructive interference between the contributions from excitation before and capture after the deflection on one hand and from both excitation and capture after the deflection on the other. From (3.12) the highvelocity behaviour is easily derived. For $v \gg Z_{\mathrm{T}}, a_{f i}^{\mathrm{R}} \sim v^{-4}$ which is much slower than the decrease of the contribution from the potential term. Thus the capture due to target recoil will become dominant for large $v$ and backward scattering angles.

\section{Numerical results}

We have calculated the transfer probability from an initially full $\mathrm{K}$ shell to the ground state of a bare projectile as

$$
P(\vartheta)=2\left|a_{f i}+a_{f i}^{\mathrm{R}}\right|^{2}
$$

with $a_{f i}$ from equation (3.11) and $a_{f i}^{\mathrm{R}}$ from equation (3.12).

Before we discuss our numerical results, it should be pointed out that while $H_{0}(s, v, x)$ is a well behaved function, the remaining part of the $x$ integrand in $a_{2}$ of equation (3.11) generally contains several (integrable) singularities, which must be taken care of. Firstly, for $s>s_{\min _{0}}$, the denominator $\Delta E-s v x+\frac{1}{2} v^{2}$ has a pole at $x=x_{0}$, which induces a logarithmic singularity in the $s$ integral as $s \rightarrow s_{\min _{0}}$. Thus, the $s$ integral should be divided at $s=s_{\min _{0}}$ for $a_{2}$. For $s>s_{\min _{0}}$ the singularity can then be removed by dividing the range of integration at $x=x_{0}$ and changing variable to $y=\ln \left|x-x_{0}\right|$.

Secondly, there is a possible square-root singularity, combined with a step function at $\left|C_{i}\right|=B$ (cf equations (A.7) and (3.11)). This occurs if the roots of the equation $\left|C_{i}\right|=B$ are in the integration interval, i.e. if $\left|x_{1,2}^{(i)}\right| \leqslant 1$, where $i=0,1$ and

$$
x_{i, 2}^{(i)}=x_{i} \cos \vartheta \pm \sqrt{1-x_{i}^{2}} \sin \vartheta
$$

In that case, one should divide the integration interval at the corresponding values of $y$. For $\vartheta \rightarrow 0, \pi$ these singularities combine to a single pole, at $x_{1}^{(i)}=x_{2}^{(i)}= \pm x_{i}$. For $\vartheta=0$, this pole just cancels the previously mentioned pole at $x_{0}$, so that equation (3.11) reduces to the straight-line transfer amplitude. For backward angles, the problem is avoided by using $y=\ln \left|x+x_{i}\right|$ as the integration variable if $\vartheta>\pi / 2$ in all terms containing $\left(C_{i}^{2}-B^{2}\right)^{1 / 2}$.

When applying the Briggs peaking approximation, it should be remembered that it only becomes exact (for s-s capture) as $v \rightarrow \infty$. For intermediate velocities, which are our main concern in the present paper, it is known to be poor for the IA (JakubaßaAmundsen and Amundsen 1980). It has been argued, however, that it should be much better for the SPB approximation than for the IA (Macek and Alston 1982). Comparison of the present results at $\vartheta=0^{\circ}$ with those based on an improved peaking approximation (Jakubaßa-Amundsen and Amundsen 1981) confirms this.

We have calculated $\mathrm{K}$ capture from $\mathrm{He}, \mathrm{C}$ and $\mathrm{Ne}$ into $\mathrm{H}$ 1s states, using Slaterscreened hydrogenic wavefunctions and experimental binding energies for the target atom. Figures 1 and 2 show the scattering angle dependence of the capture probabilities, $P(\vartheta)$, for $\mathrm{C}$ and $\mathrm{Ne}$, respectively, for impact energies in the range $0.3<E<20 \mathrm{MeV}$. It is seen that in general the dependence on $\vartheta$ is rather strong, in contrast to the IA results presented in AJA. The latter would, after correcting for the normalisation of the off-shell wavefunction in the manner of Macek and Taulbjerg (1981), be represented by a horizontal line connecting to $P\left(0^{\circ}\right)$. 


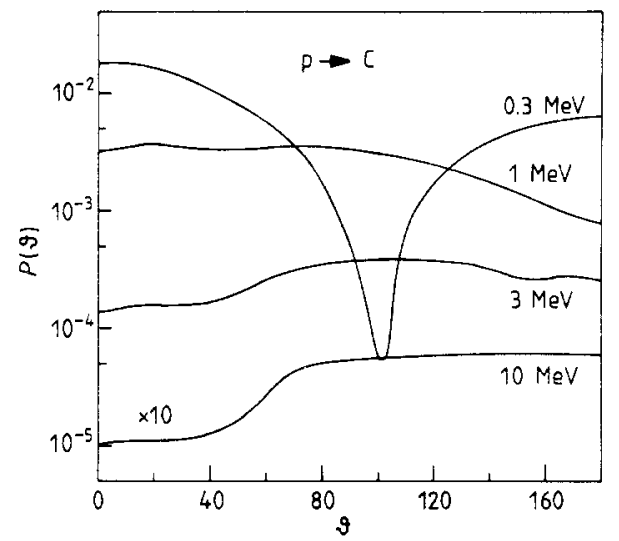

Figure 1. Capture probability from the $\mathrm{C} \mathrm{K}$ shell in collisions with protons as a function of the scattering angle $\vartheta$, for different projectile energies, calculated in the SPB approximation including projectile recoil.

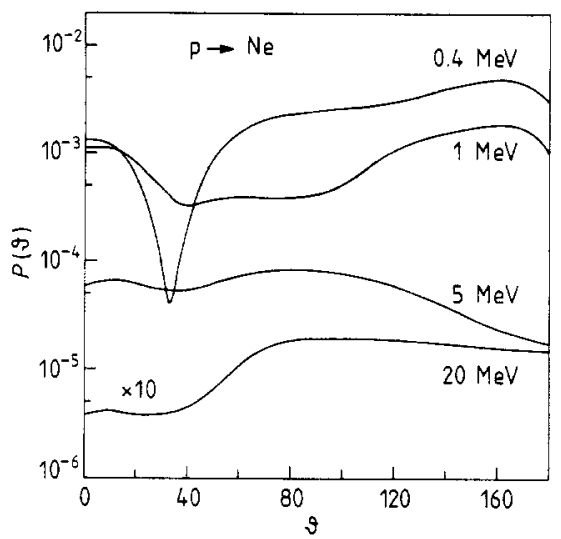

Figure 2. Capture probability from the $\mathrm{Ne} \mathrm{K}$ shell in collisions with protons (otherwise as in figure 1).

In order to investigate the importance of projectile recoil, we have performed calculations where $W_{f n}$ is replaced by $\delta_{n f}$ in equation (2.4). The resulting amplitude can then be evaluated using the same approximations and techniques as in $\S 3$, although the details are slightly different. In figure 3 we compare results of the two types of calculations for $1 \mathrm{~s}-1 \mathrm{~s}$ capture of $\mathrm{C}$ by protons at $500 \mathrm{keV}$. It is seen that even in the absence of projectile recoil, the SPB results have a strong angular dependence, in contrast to the corresponding IA results. However, it is also clear that the projectile

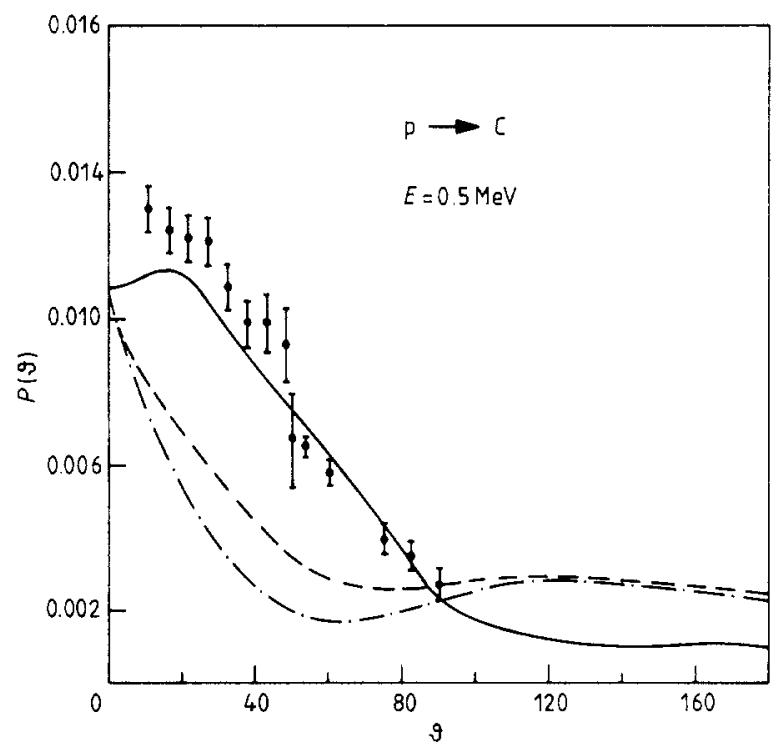

Figure 3. Probability for electron capture from $\mathrm{C}$ in collisions with $0.5 \mathrm{MeV}$ protons as a function of scattering angle $\vartheta$. The curves show SPB calculations with (full curve), without (broken curve) and partial (only intermediate 1s states, chain curve) projectile recoil, respectively. Experimental points are from Horsdal Pedersen et al $(1982 \mathrm{~b}, \mathrm{c})$. 
recoil is an important effect, as pointed out by Kocbach and Briggs (1983), and only if both effects are included, do we have a satisfactory agreement between theory and the experimental results of Horsdal Pedersen et al $(1982 \mathrm{~b}, \mathrm{c})$. Note that the experimental results are total capture probabilities for a methane target, but because of the diffuse character of the valence electrons in this system, it is assumed that these only give a minor contribution to $P(\vartheta)$ at this high collision energy.

The role of the projectile recoil can be examined in somewhat more detail by investigating contributions from various intermediate states in the sum over $n$ in equation (2.4). In particular, if it is thought that only capture to the ground state on the incoming trajectory is important, one can replace $W_{n, 1 \mathrm{~s}}$ in equation (2.4) (for $f=1 \mathrm{~s}$ ) by $W_{1 \mathrm{~s}, 1 \mathrm{~s}} \delta_{n, 1 \mathrm{~s}}$. This means that an electron captured in the $1 \mathrm{~s}$ state before the nuclear scattering has a probability of $\left|W_{1 \mathrm{~s}, 1 \mathrm{~s}}\right|^{2}$ of surviving the violent change of direction of the projectile. In particular $W_{1 \mathrm{~s}, 1 \mathrm{~s}}=0$ corresponds to the assumption that all electrons that are captured in the $1 \mathrm{~s}$ state by the projectile before the nuclear collision are lost again, and indeed $W_{1 \mathrm{~s}, \text { is }}$ tends to become very small even for moderate collision velocities and scattering angles. Again, one can carry out an analysis along the lines of $\S 3$, with minor modifications. In figure 3 we show results also of this calculation. It is seen that $P(\vartheta)$ is reduced well below the other calculations at small $\vartheta$ and if the collision energy is further increased, the reduction becomes very large at scattering angles below $60^{\circ}$. Thus, the role of the projectile recoil is not only to shake off electrons captured in the ground state on the incoming leg, but also to 'shake down' electrons captured to excited states, including the continuum, onto the final state. This latter process, in almost all cases investigated, dominates the shake-off process, so that the calculations with projectile recoil lie above those without. From an analysis of the states that can contribute to the sum in equation (2.6) for a given momentum, one finds that the important states mostly must be continuum states, so that the recoil causes a large 'shake down' of electrons that are in the continuum with respect to the projectile. An equivalent way of seeing the same thing is to evaluate $W_{n, 1 \mathrm{~s}}$ and see that in most cases the projectile recoil coupling to the continuum will be the dominating one.

In figure 4 we show the energy dependence of $P(\vartheta)$ at $\vartheta=20^{\circ}$ as a function of collision energy for $\mathrm{p}+\mathrm{C}$ for the various calculations discussed above. It is seen that for fast collisions ( $E \geqslant 400 \mathrm{keV}$ ), the SPB results including projectile recoil reproduce the experimental results for total capture by protons from $\mathrm{CH}_{4}$ very well. The theory neglecting projectile recoil entirely systematically underestimates the experimental results, while the calculations that contain projectile recoil only as a shake off of electrons captured on the incoming trajectory even show the wrong velocity dependence. For slow collisions, measurements of total capture cross sections show that capture of valence electrons cannot be neglected (Horsdal Pedersen et al 1982b), and the rise of the experimental results above the theoretical ones is consistent with an increased contribution from this process. Also notice that for these slow collisions the net result of the projectile recoil is indeed to reduce the total capture probability. This is not true for backward scattering, however.

The experimental measurements do not discriminate against capture to excited states, which we have not included in our calculations. For capture at $0^{\circ}$ scattering angle this contribution is known to be small (some 10-20\%) for velocities large enough for resonant transfer to be impossible, and this is essentially due to the narrowness of the excited projectile states in momentum space, so that the same should be true for large-angle scattering, although the angular dependence may be very different (cf AJA). The inclusion of projectile recoil should not change this dramatically, since the effective 


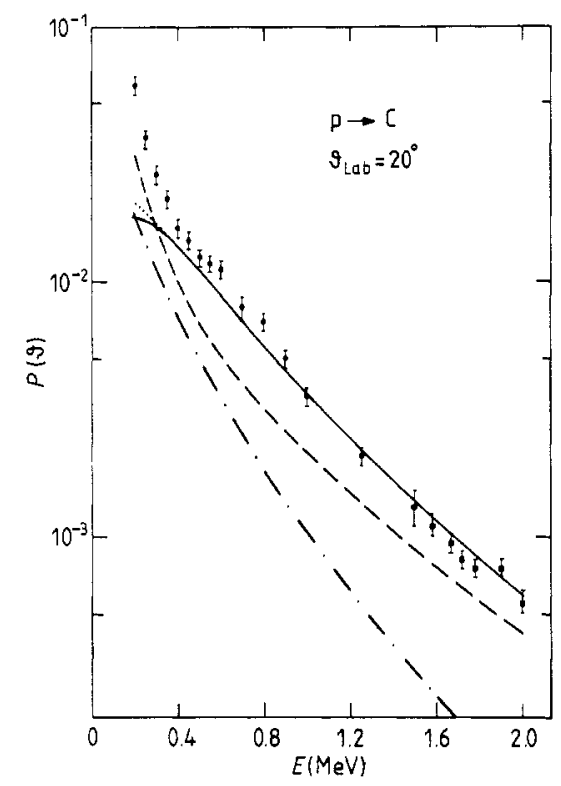

Figure 4. Probability for electron capture from $\mathrm{C}$ in collision with protons at a scattering angle of $20^{\circ}$, as a function of projectile energy. The full, broken and chain curves and experimental points are as for figure 3 . The dotted curve shows the effect of adding recoil shake up to excited projectile states to the full SPB calculations. See text for details. The experimental results above $1.5 \mathrm{MeV}$ are for $\vartheta=15^{\circ}$.

projectile state of equation (2.7) is only shifted in momentum space by $\Delta v$ with respect to the wavefunction in the absence of recoil, and this does not influence its width. A particular contribution to the capture to excited states can also be included easily, if it is assumed that direct capture to excited states is very small, but if the electron is initially captured into the $1 \mathrm{~s}$ state, it has a significant probability of being shaken up into some other bound state by the recoil. Since the final state is different, this contribution should be added incoherently to $P(\vartheta)$, and it is given by

$$
\begin{aligned}
P^{\mathrm{su}}(\boldsymbol{\vartheta}) & =2 \underset{\substack{f \neq 1 \mathrm{~s} \\
\boldsymbol{E}_{f}<0}}{\sum_{f i}\left|a_{f i}^{<}\right|^{2}\left|W_{f, 1 \mathrm{~s}}\right|^{2}} \\
& =2\left|a_{f i}^{<}\right|^{2}\left(1-\left|W_{1 \mathrm{~s}, 1 \mathrm{~s}}\right|^{2}-\int \mathrm{d} \boldsymbol{k}_{f}\left|W_{\boldsymbol{k}_{f}, 1 \mathrm{~s}}\right|^{2}\right)
\end{aligned}
$$

where we have used the completeness of the projectile states. Here $a_{f i}^{<}$is the amplitude for capture on the incoming trajectory only, obtained from equation (2.4) restricted to $t \leqslant t^{\prime}<0$. This can also be evaluated with the methods of $\S 3$, while $W_{k, 15}$ can be found analytically. In figure 4 we show the contribution from this term to $P\left(20^{\circ}\right)$. It is of some importance only at low collision velocities and at rather small scattering angles since, when the recoil becomes stronger, the electrons will be shaken off into the continuum. This simple calculation confirms that the inclusion of projectile recoil will not dramatically change the importance of capture to excited states.

In contrast to the projectile recoil, the target recoil term, i.e. the contribution of the recoil to excitation of the target, given by equation (3.12), shows a very simple behaviour. It is always important at backward scattering angles, becoming dominant 
as the collision energy is increased, in accordance with the discussion following equation (3.12). At intermediate and slow collisions it may either increase or decrease the transfer probability, depending on the phase relative to the direct excitation amplitude, an effect also known from ionisation theories (Amundsen 1978).

The calculations we have made generally show some tendency for structures in $P(\vartheta)$ to appear around $\vartheta=60^{\circ}$ (cf figures 1 and 2 ). This is in accordance with the ideas of Horsdal Pedersen and Rasmussen (1983), and also with the theory of critical scattering angles at asymptotically large velocities (Dettmann and Leibfried 1969).

We have also calculated $P(\vartheta)$ for proton impact on $\mathrm{He}$ at $E=200$ and $400 \mathrm{keV}$. In these cases one is of course certain that capture is from a $1 \mathrm{~s}$ state, on the other hand the use of a perturbative approach and hydrogenic wavefunctions is not very well justified. It turns out that $P(\vartheta)$ is rather constant, except a slow increase for $\vartheta>70^{\circ}$. This is in reasonable agreement with the experimental results available, for $\vartheta \leqslant 50^{\circ}$ (Horsdal Pedersen et al 1982a). For the higher collision energy, even the magnitude is predicted correctly, within $30 \%$.

Finally it should be pointed out that although the agreement between the SPB calculations and the available experimental results is very satisfactory, there is still room for further theoretical improvements. Perhaps most importantly, the carbon $\mathrm{K}$ shell is not very well described by screened hydrogenic wavefunctions, but it is also not clear how well the off-shell states are represented by renormalised on-shell ones (equation (3.1)), and if the system $\mathrm{p}+\mathrm{C}$ is asymmetric enough to completely exclude significant contributions from higher order terms in $\left(V_{\mathrm{P}}+V_{\mathrm{R}}\right)$ in the SPB expansion.

\section{Conclusion}

We have extended the strong-potential Born approximation for charge transfer to the case of large-angle projectile scattering. Calculations of $1 \mathrm{~s}-1 \mathrm{~s}$ capture, including recoil effects on the projectile states, using the usual multiplicative renormalisation of the intermediate off-shell state, Briggs' peaking approximation in the intermediate momentum integrals and hydrogenic wavefunctions, show a strong scattering-angle dependence of the transfer probability in most cases investigated. This strong scattering-angle dependence has two causes, which generally are about equally important. The first is that the SPB, except for forward scattering, allows for a time delay between the excitation of the target atom and the capture of the electron by the projectile. This amplitude is not present in the IA. The second cause is the inclusion of projectile recoil non-perturbatively, which induces a scattering angle dependence for $1 \mathrm{~s}-1 \mathrm{~s}$ capture also in the IA.

The present calculations show a clearly improved agreement with experimental data for $\mathrm{K}$ capture from $\mathrm{CH}_{4}$ to protons at intermediate velocities. For a more stringent test of the theory, direct measurements of the $1 \mathrm{~s}-1 \mathrm{~s}$ capture probability at large scattering angles in asymmetric collisions would be very welcome.

\section{Acknowledgments}

We thank E Horsdal Pedersen for stimulating discussions and access to unpublished experimental data and L Kocbach for informing us of his work prior to publication. 
This work was partly carried out while one of the authors (PAA) was an Alexander von Humboldt fellow at the Technische Universität München. The other (DHJ-A) thanks GSI Darmstadt and NORDITA for financial support.

\section{Appendix}

In this appendix we evaluate the $\boldsymbol{k}$ and one of the $s$ integrals in equation (3.8). We need the formulae $\left(\eta=Z_{\mathrm{T}} / v\right)$ :

$$
\begin{gathered}
\int \mathrm{d} \boldsymbol{k}\left[Z_{\mathrm{P}}^{2}+\left(\boldsymbol{k}-v \boldsymbol{e}_{z}\right)^{2}\right]^{-2-\mathrm{i} \eta}=\frac{\pi^{3 / 2}}{Z_{\mathrm{P}}^{1+2 \mathrm{i} \eta}} \frac{\Gamma\left(\frac{1}{2}+\mathrm{i} \eta\right)}{\Gamma(2+\mathrm{i} \eta)} \\
\int \mathrm{d} \boldsymbol{k} \frac{\left(A+\mathrm{i} \varepsilon-\frac{1}{2} k^{2}\right)^{-\mathrm{i} \eta}}{\left[Z_{\mathrm{P}}^{2}+\left(\boldsymbol{k}-v \boldsymbol{e}_{z}\right)^{2}\right]^{2}} \\
=4 \pi \int_{0}^{\infty} k^{2} \mathrm{~d} k \frac{\left(A+\mathrm{i} \varepsilon-\frac{1}{2} k^{2}\right)^{-\mathrm{i} \eta}}{\left(Z_{\mathrm{P}}^{2}+k^{2}+v^{2}\right)^{2}-4 k^{2} v^{2}} \\
=\frac{\pi^{2}}{2 Z_{\mathrm{P}} v}\left(\frac{v-\mathrm{i} Z_{\mathrm{P}}}{\left[A+\mathrm{i} \varepsilon-\frac{1}{2}\left(v-\mathrm{i} Z_{\mathrm{P}}\right)^{2}\right]^{\mathrm{i} \eta}}+\frac{v+\mathrm{i} Z_{\mathrm{P}}}{\left[A+\mathrm{i} \varepsilon-\frac{1}{2}\left(v+\mathrm{i} Z_{\mathrm{P}}\right)^{2}\right]^{\mathrm{i} \eta}}\right)+\mathrm{O}(\varepsilon) .
\end{gathered}
$$

The last integral can be done by the calculus of residues by extending the range of integration to $k=-\infty$, keeping below the cut in the complex $k$ plane extending from $k=-\sqrt{2 A}-i \varepsilon$ to $\sqrt{2 A}+i \varepsilon$. This ensures the correct phase, and the contribution from avoiding the leftmost branch point is only $O(\varepsilon)$. The contour can then be completed with an infinite semicircle in the lower half-plane, and the residues taken at the poles at $k=\mp v-\mathrm{i} Z_{\mathrm{P}}$.

The $\boldsymbol{k}$ integral from the last term in (3.8) is obtained as

$$
\begin{aligned}
\int \mathrm{d} \boldsymbol{k} \frac{\left[Z_{\mathrm{P}}^{2}+\left(\boldsymbol{k}-v \boldsymbol{e}_{z}\right)^{2}\right]^{-\mathrm{i} \eta}}{\left[Z_{\mathrm{P}}^{2}+\left(\boldsymbol{k}-\tilde{\boldsymbol{v}}_{+}\right)^{2}\right]^{2}} \\
=4 \pi \int_{0}^{\infty} q^{2} \mathrm{~d} q \frac{\left(Z_{\mathrm{P}}^{2}+q^{2}\right)^{-\mathrm{i} \eta}}{q^{4}+2 q^{2} Z_{\mathrm{P}}^{2}\left(1-\xi^{2}\right)+Z_{\mathrm{P}}^{2}\left(1+\xi^{2}\right)^{2}} \\
=\frac{2 \pi}{Z_{\mathrm{P}}^{1+2 \mathrm{i} \eta}\left(t_{+}-t_{-}\right)} \int_{0}^{\infty} \mathrm{d} t t^{1 / 2}(1+t)^{-\mathrm{i} \eta}\left(\frac{1}{t-t_{+}}-\frac{1}{t-t_{-}}\right) \\
=\frac{\mathrm{i} \pi^{3 / 2}}{4 Z_{\mathrm{P}}^{1+2 i \eta} \xi} \frac{\Gamma\left(-\frac{1}{2}+\mathrm{i} \eta\right)}{\Gamma(1+\mathrm{i} \eta)}\left[\frac{1}{t_{+}}{ }_{2} F_{1}\left(1, \frac{3}{2} ; 1+\mathrm{i} \eta ; 1+\frac{1}{t_{+}}\right)\right. \\
\left.\quad-\frac{1}{t_{-}}{ }_{2} F_{1}\left(1, \frac{3}{2} ; 1+\mathrm{i} \eta ; 1+\frac{1}{t_{-}}\right)\right]
\end{aligned}
$$

where we used the subsequent substitutions $\boldsymbol{q}=\boldsymbol{k}-\boldsymbol{v} \boldsymbol{e}_{z}$ and $t=q^{2} / Z_{\mathrm{P} .}^{2}{ }_{2} F_{1}$ is a hypergeometric function and we use the abbreviations $\xi^{2}=2 v^{2}(1-\cos \vartheta) / Z_{\mathrm{P}}^{2}$ and $t_{ \pm}=$ $\xi^{2}-1 \odot 2 \mathrm{i} \xi$.

The next step is the evaluation of the $s$ integrals in equation (3.8). Let us first consider the two terms proportional to the $\delta$ functions. In the three-dimensional integral over $s$, the integral over $x=\cos \vartheta_{s}$ can be done trivially; but since the peaking approximation has selected different values of $\boldsymbol{k}, \boldsymbol{x}$ and the minimum momentum transfer $s_{\min }$ become different in the two terms. When doing the integral over the 
azimuthal angle $\varphi_{s}$, one must keep in mind that in the last term, the matrix element has become dependent on $\varphi_{s}$ from the replacement of $\boldsymbol{k}$ by $\tilde{\boldsymbol{v}}_{+}$. Still, this integral can be done with the help of

$$
\begin{gathered}
\int_{0}^{2 \pi} \mathrm{d} \varphi_{s}\left(\lambda_{1}-\mu_{1} \cos \varphi_{s}\right)^{-n+\mathrm{i} \eta}=2 \pi\left(\lambda_{1}+\mu_{1}\right)^{-n+\mathrm{i} \eta}{ }_{2} F_{1}\left(n-\mathrm{i} \eta, \frac{1}{2} ; 1 ; \frac{2 \mu_{1}}{\lambda_{1}+\mu_{1}}\right) \\
\lambda_{1}=Z_{\mathrm{T}}^{2}+s^{2}+v^{2}-2 s x_{1} v \cos \vartheta \\
\mu_{1}=2 s\left(1-x_{1}^{2}\right)^{1 / 2} v \sin \vartheta \\
x_{1}=\left(\Delta E+v^{2} \cos \vartheta-\frac{1}{2} v^{2}\right) / s v
\end{gathered}
$$

with $n=1,2$. For the other term, no $\varphi_{s}$ dependence is present and the $\varphi_{s}$ integral yields $2 \pi$. For the remaining terms of equation (3.8), we can only do one integral, the one over $\varphi_{s}$, which enters non-trivially through $\tilde{s}_{x}$ in three of the terms. There are two types of these integrals, namely

$$
\begin{aligned}
& P \int_{0}^{2 \pi} \mathrm{d} \varphi_{s} \frac{1}{C-B \cos \varphi_{s}}=\frac{2 \pi \operatorname{sign} C}{\left(C^{2}-B^{2}\right)^{1 / 2}} \theta(|C|-B) \\
& C=\Delta E-s x v \cos \theta+\frac{1}{2} v^{2} \\
& B=s v\left(1-x^{2}\right)^{1 / 2} \sin \vartheta
\end{aligned}
$$

and $(i=0,1)$

$$
\begin{gathered}
P \int_{0}^{2 \pi} \mathrm{d} \varphi_{s} \frac{\left(A_{ \pm}+B \cos \varphi_{s}\right)^{-\mathrm{i} \eta}}{C_{i}-B \cos \varphi_{s}}=2 \pi P \frac{\left(A_{ \pm}-B\right)^{-\mathrm{i} \eta}}{C_{i}+B} F_{1}\left(\frac{1}{2} ; \mathrm{i} \eta, 1 ; 1 ; \frac{2 B}{B-A_{ \pm}}, \frac{2 B}{C_{i}+B}\right) \\
A_{ \pm}=E_{\mathrm{i}}^{\mathrm{T}}+s v x \cos \vartheta-\frac{1}{2}\left(v \pm \mathrm{i} Z_{\mathrm{P}}\right)^{2}+\mathrm{i} \varepsilon \\
C_{0}=C \\
C_{1}=\Delta E-s x v \cos \vartheta+v^{2} \cos \vartheta-\frac{1}{2} v^{2} .
\end{gathered}
$$

In writing down equation (A.5) we have explicitly used the implied principal value prescription stated after equation (3.6), while the $P$ in front of the Appell hypergeometric function $F_{1}$ (for the definition of this, see Gradshteyn and Ryzhik (1980), $\S 9.18$ ), means that one should take the average of this function across a branch cut, when necessary.

However, the calculation of $F_{1}$ in (A.6) is rather time consuming. We have therefore applied an approximation which was introduced in $\mathrm{AJA}$, based on the fact that for $\left|C_{\mathrm{i}}\right| \geqslant B$, the integrand in (A.6) is strongly peaked at $\cos \varphi_{s}= \pm 1$ for $C_{i} \gtrless 0$, while for $\left|C_{\mathrm{i}}\right|<B$, the contributions from both sides of the singularity at $\cos \varphi_{s}=C_{i} / B$ tend to cancel each other. Taking the term $\left(A_{ \pm}+B \cos \varphi_{s}\right)^{-\mathrm{i} \eta}$ outside the integral at $\cos \varphi_{s}=$ \pm 1 , one then finds

$$
\begin{gathered}
P \int_{0}^{2 \pi} \mathrm{d} \varphi_{s} \frac{\left(A_{ \pm}+B \cos \varphi_{s}\right)^{-\mathrm{i} \eta}}{C_{i}-B \cos \varphi_{s}} \simeq \frac{2 \pi F_{\mathrm{i}}\left(A_{ \pm}\right)}{\left(C_{i}^{2}-B^{2}\right)^{1 / 2}} \\
F_{i}(A)=\left\{\begin{array}{cl}
(A+B)^{-\mathrm{i} \eta} & C_{i} \geqslant B \\
-(A-B)^{-\mathrm{i} \eta} & C_{i} \leqslant-B \\
0 & \left|C_{i}\right|<B .
\end{array}\right.
\end{gathered}
$$


In spite of its apparent crude nature, this approximation is exact for $\left|C_{i}\right|=B$ and also for $B=0$, i.e. for $\vartheta=0$ and $\vartheta=\pi$. Inserting these results in equation (3.8), we arrive at equation (3.11).

\section{References}

Abramowitz M and Stegun I A 1965 Handbook of Mathematical Functions (New York: Dover) ch 6 Amundsen P A 1978 J. Phys. B. At. Mol. Phys. $113197-220$

Amundsen P A and Jakubaßa-Amundsen D H 1982 Phys. Scr. 26 155-62

Briggs J S 1977 J. Phys. B: At. Mol. Phys. 10 3075-89

Chen J C Y and Chen A C 1972 Adv. At. Mol. Phys. 8 71-129

Ciocchetti G and Molinari A 1965 Nuovo Cim. B 40 69-86

Dettman K 1971 Springer Tracts in Modern Physics vol 58 pp 119-208

Dettman K and Leibfried G 1969 Z. Phys. 218 1-24

Gradshteyn I S and Ryzhik I M 1980 Tables of Integrals, Series and Products (New York: Academic) (corrected and enlarged edn)

Horsdal Pedersen E, Loftager P and Rasmussen J L 1982a J. Phys. B: At. Mol. Phys. 15 2461-71

1982b J. Phys. B: At. Mol. Phys. 15 4423-36

$1982 \mathrm{c}$ private communication

Horsdal Pedersen E and Rasmussen J L 1983 IEEE Trans. Nucl. Sci. NS-30 891-4

Jakubaßa-Amundsen D H and Amundsen P A 1980 Z. Phys. A 297 203-14

1981 J. Phys. B: At. Mol. Phys, 14 L705-11

Kocbach L and Briggs J S 1983 Proc. 13th Int. Conf. on the Physics of Electronic and Atomic Collisions, Berlin ed J Eichler et al (Amsterdam: North-Holland) Abstracts p 498 and in preparation

McDowell M R C 1961 Proc. R. Soc. A $264277-88$

McDowell M R C and Coleman J P 1970 Introduction to the Theory of Ion-Atom Collisions (Amsterdam: North-Holland)

Macek J and Alston S 1982 Phys. Rev. A 26 250-70

Macek J and Shakeshaft R 1980 Phys. Rev. A 22 1441-6

Macek J and Taulbjerg K 1981 Phys. Rev. Lett. 46 170-4 